\title{
A Histologically Proven Case of Autoimmune Hepatitis with Eosinophilic Fasciitis
}

\author{
Shohei Asada ${ }^{1}$, Akitoshi Douhara ${ }^{1}$, Koji Murata ${ }^{1}$, Koji $_{\text {Yanase }}{ }^{1}$, \\ Masahiro Tsutsumi ${ }^{2}$ and Hitoshi Yoshiji ${ }^{3}$
}

\begin{abstract}
:
Both autoimmune hepatitis (AIH) and eosinophilic fasciitis (EF) are known to be complicated by other autoimmune diseases. However, AIH complicated by EF has never been reported. We experienced a 58-yearold man with AIH complicated by EF. He was admitted to our hospital with acute hepatic injury and edema of the legs in April 201X. The etiologies of these symptoms were histologically proven as AIH and EF. The administration of prednisolone (PSL) drastically improved his liver injury and edema of the legs. When we make a diagnosis of $\mathrm{AIH}$, we should carefully evaluate the physical findings, including the appearance of the legs, in order to detect other coexisting autoimmune diseases.
\end{abstract}

Key words: eosinophilic fasciitis, autoimmune hepatitis, autoimmune disease, prednisolone

(Intern Med 58: 667-673, 2019)

(DOI: 10.2169/internalmedicine.1299-18)

\section{Introduction}

Autoimmune hepatitis (AIH) is known to be often complicated by other autoimmune diseases, such as chronic thyroiditis, Sjögren's syndrome, rheumatoid arthritis, systemic lupus erythematosus, primary biliary cholangitis, and Grave's disease (1). Eosinophilic fasciitis (EF) is a rare scleroderma-like connective tissue disease characterized by symptoms of edema of the limbs and inflammatory cell infiltration localized in the fascia. Classified as an autoimmune disorder, EF was first described in 1974 by Shulman (2) and is also known to be complicated by other autoimmune diseases, such as scleroderma, polymyositis, hypereosinophilic syndrome, and Churg-Strauss vasculitis (3).

We herein report a histologically proven case of AIH complicated by EF. These diseases have never been reported to be complicated with each other. In our case, prednisolone administration was effective for the improvement of both $\mathrm{AIH}$ and EF. At the time a diagnosis of AIH is made, we should examine the general findings, including the appearance of the legs, to detect complications of other autoimmune diseases, such as EF.

\section{Case Report}

A 58-year-old man visited our hospital complaining of bilateral leg edema. He had a history of nephrotic syndrome at eight years of age, but had no recurrence. He never had a history of liver dysfunction. He reported no habitual drug use, allergies, cigarette smoking, or alcohol consumption.

His first laboratory test (Table 1) showed a slight increase in eosinophils, C-reactive protein (CRP), and aspartate aminotransferase (AST), as well as mild hypoalbuminemia. However, the cause of his leg edema was still not clear despite the performance of urinary and blood tests, upper and lower gastrointestinal endoscopies, and abdominal ultrasound sonography (US). Six months after his first visit to our hospital, he returned complaining of malaise and jaundice with gradual progression beginning one month earlier. His height was $172 \mathrm{~cm}$, his body weight was $58.6 \mathrm{~kg}$, and his body mass index was $19.8 \mathrm{~kg} / \mathrm{m}^{2}$. Vital signs were not appreciable. His physical findings showed icteric conjunctiva and bilateral leg edema. His laboratory tests (Table 2) showed remarkable liver dysfunction, jaundice, deterioration of hepatic functional reserve, and high hepatic fibrosis

${ }^{1}$ Department of Internal Medicine, Saiseikai Chuwa Hospital, Japan, ${ }^{2}$ Department of Pathology, Saiseikai Chuwa Hospital, Japan and ${ }^{3}$ Third department of Internal Medicine, Nara Medical University, Japan

Received: April 2, 2018; Accepted: August 16, 2018; Advance Publication by J-STAGE: October 17, 2018

Correspondence to Akitoshi Douhara, aki-do@hotmail.co.jp 
Table 1. Laboratory Data on His First Visit.

\begin{tabular}{lclr}
\hline Hematology & & AST & $42 \mathrm{IU} / \mathrm{L}$ \\
WBC & $7,900 / \mu \mathrm{L}$ & ALT & $28 \mathrm{IU} / \mathrm{L}$ \\
Neu & $59.9 \%$ & ALP & $249 \mathrm{IU} / \mathrm{L}$ \\
Lym & $27.6 \%$ & $\gamma \mathrm{GTP}$ & $24 \mathrm{IU} / \mathrm{L}$ \\
Mono & $3.5 \%$ & LDH & $315 \mathrm{IU} / \mathrm{L}$ \\
Eos & $8.7 \%$ & ChE & $147 \mathrm{IU} / \mathrm{L}$ \\
Baso & $0.3 \%$ & Glu & $93 \mathrm{mg} / \mathrm{dL}$ \\
RBC & $433 \times 10^{4} / \mu \mathrm{L}$ & CRP & $0.35 \mathrm{mg} / \mathrm{dL}$ \\
Platelet & $37.1 \times 10^{4} / \mu \mathrm{L}$ & T-cho & $134 \mathrm{mg} / \mathrm{dL}$ \\
Chemistry & & TG & $102 \mathrm{mg} / \mathrm{dL}$ \\
TP & $6.2 \mathrm{~g} / \mathrm{dL}$ & TSH & $1.2 \mu \mathrm{IU} / \mathrm{mL}$ \\
Alb & $3.1 \mathrm{~g} / \mathrm{dL}$ & FT3 & $2.94 \mathrm{pg} / \mathrm{mL}$ \\
UN & $15 \mathrm{mg} / \mathrm{dL}$ & FT4 & $0.94 \mathrm{ng} / \mathrm{dL}$ \\
Cre & $0.99 \mathrm{mg} / \mathrm{dL}$ & BNP & $17.5 \mathrm{pg} / \mathrm{mL}$ \\
UA & $6.6 \mathrm{mg} / \mathrm{dL}$ & Anti scleroderma 70 antibody & Negative \\
Na & $140 \mathrm{mEq} / \mathrm{L}$ & Anti ribonucleoprotein antibody & Negative \\
K & $4.3 \mathrm{mEq} / \mathrm{L}$ & Anti centromere antibody & Negative \\
Cl & $107 \mathrm{mEq} / \mathrm{L}$ & Urine protein & Negative \\
T-bil & $0.4 \mathrm{mg} / \mathrm{dL}$ & Urine occult blood & Negative \\
\hline
\end{tabular}

WBC: White blood cell, Neu: Neutrophil, Lym: Lymphocyte, Mono: Monocyte, Eos: Eosinophil, Baso: Basophil, RBC: Red blood cell, TP: Total protein, Alb: Albumin, UN: Urea nitrogen, Cre: Creatinine, UA: Uric acid, Na: Sodium, K: Potassium, Cl: Chlorine, T-bil: Total bilirubin, AST: Aspartate aminotransferase, ALT: Alanine aminotransferase, ALP: Alkaline Phosphatase, yGTP: $\gamma$-glutamyltransferase, LDH: Lactate dehydrogenase, ChE: Cholinesterase, Glu: Glucose, CRP: Creactive protein, T-cho: Total cholesterol, TG: Triglyceride, TSH: Thyroid stimulating hormone, FT3: Free triiodothyronine, FT4: Free thyroxine, BNP: Brain natriuretic peptide

Table 2. Laboratory Data Six Months Later.

\begin{tabular}{lclr}
\hline hematology & & LDH & $294 \mathrm{IU} / \mathrm{L}$ \\
WBC & $4,800 / \mu \mathrm{L}$ & ChE & $64 \mathrm{IU} / \mathrm{L}$ \\
Neu & $70.5 \%$ & CRP & $0.97 \mathrm{mg} / \mathrm{dL}$ \\
Lym & $22.0 \%$ & Type 4 collagen $7 \mathrm{~S}$ & $18 \mathrm{ng} / \mathrm{mL}$ \\
Mono & $6.0 \%$ & Hyaluronic acid & $1,790 \mathrm{ng} / \mathrm{mL}$ \\
Eos & $1.0 \%$ & Procollagen 3 peptide & $43.8 \mathrm{ng} / \mathrm{mL}$ \\
Baso & $0.5 \%$ & M2BPGi & $19.26 \mathrm{C.O} . \mathrm{I}$. \\
RBC & $380 \times 10^{4} / \mu \mathrm{L}$ & Aldolase & $12.2 \mathrm{U} / \mathrm{L}$ \\
Platelet & $17.8 \times 10^{4} / \mu \mathrm{L}$ & IgG & $2,858 \mathrm{mg} / \mathrm{dL}$ \\
Chemistry & & IgA & $303 \mathrm{mg} / \mathrm{dL}$ \\
TP & $6.5 \mathrm{~g} / \mathrm{dL}$ & IgM & $135 \mathrm{mg} / \mathrm{dL}$ \\
Alb & $2.3 \mathrm{~g} / \mathrm{dL}$ & IgE & $1,230 \mathrm{IU} / \mathrm{mL}$ \\
UN & $8.7 \mathrm{mg} / \mathrm{dL}$ & Anti nuclear antibody & 40 \\
Cre & $0.72 \mathrm{mg} / \mathrm{dL}$ & & Homogenous and speckled type \\
T-bil & $7.5 \mathrm{mg} / \mathrm{dL}$ & Anti liver/kidney microsome type1 antibody & Negative \\
D-bil & $6.0 \mathrm{mg} / \mathrm{dL}$ & Anti mitochondrial M2 antibody & Negative \\
AST & $597 \mathrm{IU} / \mathrm{L}$ & IgM class cytomegalovirus antibody & Negative \\
ALT & $210 \mathrm{IU} / \mathrm{L}$ & Epstein Barr virus capsid antigen IgM & Negative \\
ALP & $1,089 \mathrm{IU} / \mathrm{L}$ & HBs Ag & Negative \\
rGTP & $213 \mathrm{IU} / \mathrm{L}$ & HCV Ab & Negative \\
\hline
\end{tabular}

WBC: White blood cell, Neu: Neutrophil, Lym: Lymphocyte, Mono: Monocyte, Eos: Eosinophil, Baso: Basophil, RBC: Red blood cell, TP: Total protein, Alb: Albumin, UN: Urea nitrogen, Cre: Creatinine, T-bil: Total bilirubin, D-bil: Direct bilirubin, AST: Aspartate aminotransferase, ALT: Alanine aminotransferase, ALP: Alkaline Phosphatase, $\gamma$ GTP: $\gamma$-glutamyltransferase, LDH: Lactate dehydrogenase, ChE: Cholinesterase, CRP: Creactive protein, M2BPGi: Mac-2 binding protein glycan isomer, C.O.I: cut off index, IgG: Immunoglobulin G, IgA: Immunoglobulin A, IgM: Immunoglobulin M, IgE: Immunoglobulin E, HBs Ag: Hepatitis B virus surface antigen, $\mathrm{HCV} \mathrm{Ab}$ : Hepatitis $\mathrm{C}$ virus antibody 


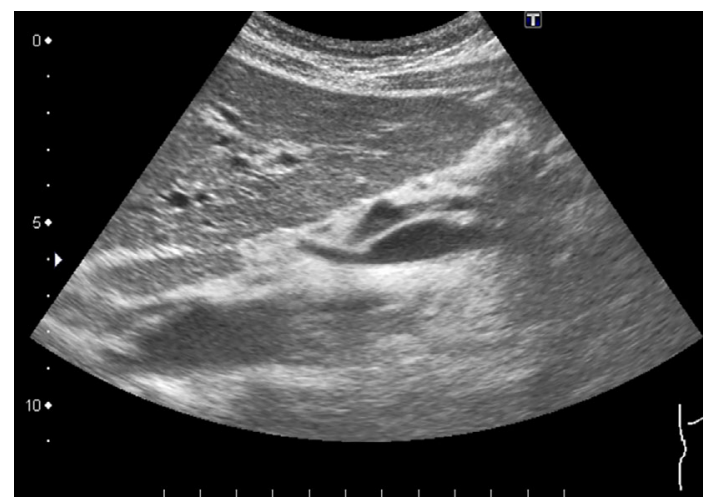

Figure 1. Abdominal ultrasonography showed chronic hepatic disorder findings (irregular surface, dull edge and heterogenous internal echo).

markers such as Type 4 collagen 7S and Mac-2 binding protein glycan isomer (M2BPGi). Hepatitis virus markers were all negative, and immunoglobulin $\mathrm{G}(\mathrm{IgG})$ and anti-nuclear antibody levels were elevated. Abdominal US (Fig. 1) revealed an irregular surface, dull edge, and a heterogenous internal echo of the liver, as well as a slightly swollen spleen. Furthermore, abdominal contrast-enhanced computed tomography (CT) (Fig. 2) showed periportal low density of the liver and an edematous gallbladder wall. These findings suggested acute on chronic hepatitis.

Based on these laboratory and imaging findings, we suspected the etiology of the liver injury to be autoimmune hepatitis. To clarify the etiology of liver dysfunction histologically, a liver biopsy was performed on the 14th hospital day. Hematoxylin and Eosin (H\&E) staining of the liver biopsy specimen revealed remarkable inflammatory cell infiltration in the portal area, interface hepatitis in the lowpower field (Fig. 3A), rosette formation (Fig. 3B), emperipolesis (Fig. 3C), and plasma cell infiltration in the highpower field (Fig. 3D). Masson trichrome staining of the liver biopsy specimen revealed fibrosis localized in the portal area (Fig. 3E). The international AIH group score in our case was 18 points, which indicated definite AIH. From these findings, we concluded that his liver injury was indicative of typical AIH.

In addition, we suspected that hypoalbuminemia due to liver dysfunction was what had caused the leg edema on admission. However, after careful observation by a dermatologist, his lower limbs revealed an "orange-peel-like appearance," which is a typical physical finding of EF (Fig. 4). Because EF was suspected based on the physical findings, additional examinations of magnetic resonance imaging (MRI) and an en bloc biopsy of the lower limbs were performed. Fat-suppressed T2-weighted MRI showed that the fascia of the lower limbs was thickening and changing to a higher intensity (Fig. 5). In contrast, the muscles of the lower legs were normal on MRI. H\&E staining of the en bloc biopsy specimen showed thickening and edema of the fascia, hyperplasia and degeneration of collagenous fiber, and inflammatory cell infiltration, including eosinophils, in

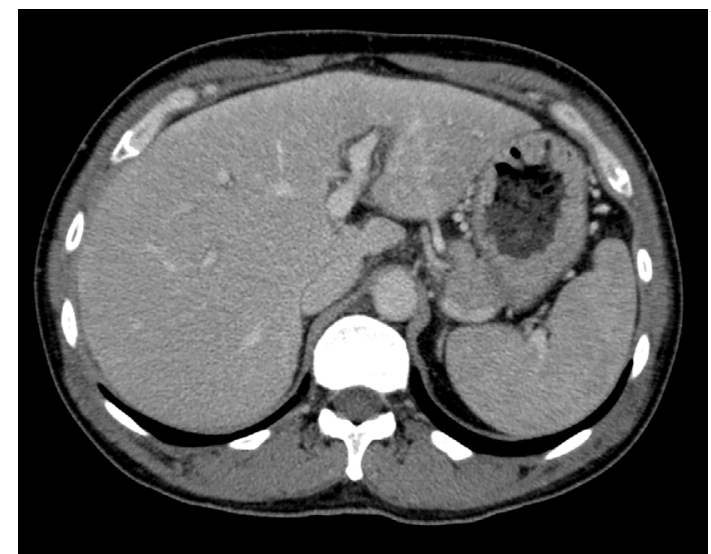

Figure 2. Abdominal contrast-enhanced CT showed periportal low density and edematous gallbladder wall, suggesting acute hepatitis.

the fascia. The muscle and subcutaneous tissues were normal (Fig. 6). Based on these findings, we finally made a diagnosis of $\mathrm{EF}$ of the legs.

For the treatment of both $\mathrm{AIH}$ and $\mathrm{EF}$, prednisolone (PSL) $40 \mathrm{mg} / \mathrm{day}$ was started on the 16th hospital day. After that, his liver dysfunction gradually improved. His leg edema and serum aldolase, which is a known biomarker of muscular disease, including EF, also slowly improved. The dosage of PSL was gradually decreased. He was discharged on the $51 \mathrm{st}$ hospital day. PSL $10 \mathrm{mg} /$ day is being continued for maintenance therapy. His liver function remains in the normal range, and his leg edema has almost completely disappeared (Fig. 7).

\section{Discussion}

Our case suggested two clinical issues. First, EF can be complicated by AIH. Second, EF complicated by chronic liver disease can be missed.

In terms of the pathogenesis, EF can be complicated by AIH. AIH is characterized by hypergammaglobulinemia, seropositivity for autoantibodies, interface hepatitis on histology, and response to immunosuppressive treatment (4). The pathogenesis of AIH is not completely clear, but it is thought to have genetic factors and features of immunological disorders, such as impairment of regulatory T-cells and the breakdown of immunological tolerance against selfantigens $(4,5)$. AIH is known to be complicated by other autoimmune diseases, such as chronic thyroiditis, Sjögren's syndrome, and rheumatoid arthritis (1). AIH is substantially improved by immunosuppressive treatment. $\mathrm{EF}$ is a scleroderma-like disease characterized by swelling and edematous extremities, hypergammaglobulinemia, and thickened and fibrotic fascia with the infiltration of inflammatory cells, lymphocytes, plasma cells, histiocytes, and eosinophils (6). Although the pathogenesis of EF is not clear, EF is also improved by immunosuppressive treatments, such as corticosteroids and methotrexate (7), suggesting that it is an 
A

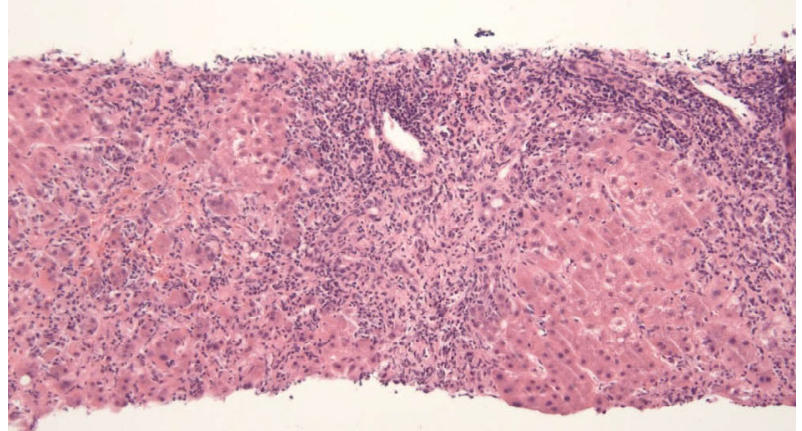

$\mathrm{B}$

C

$\mathrm{D}$
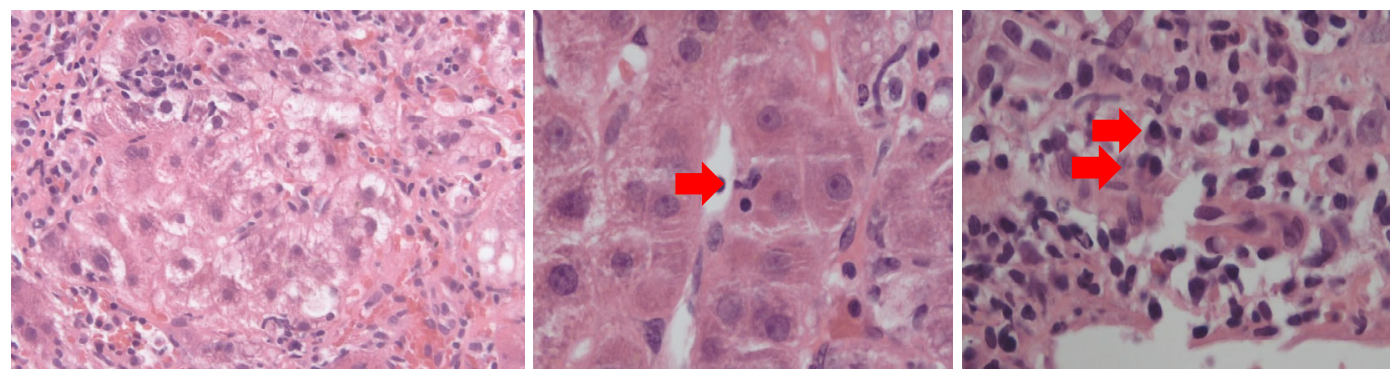

$\mathrm{E}$

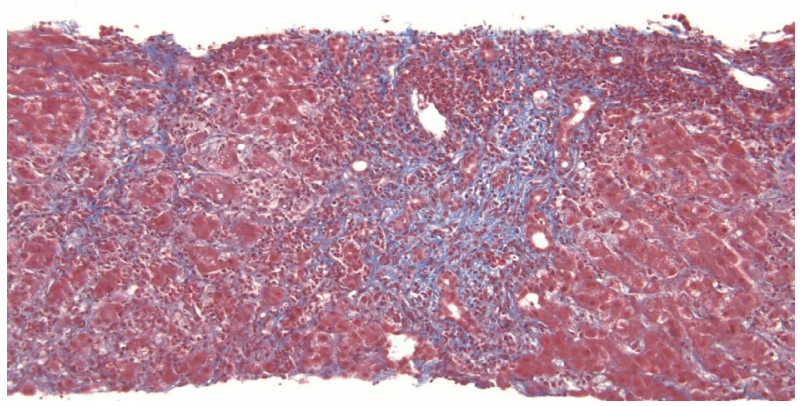

Figure 3. Hematoxylin and Eosin (H\&E) staining and Masson trichrome staining of a liver biopsy specimen. (A) Remarkable inflammatory cell infiltration at the portal area and interface hepatitis (H\&E staining, 40-fold). (B) Rosetta formation (H\&E staining, 200-fold). (C) Emperipolesis (arrow) (H\&E staining, 200-fold). (D) Plasma cell infiltration (arrow) (H\&E staining, 400-fold). (E) Fibrosis localized in the portal area (Masson trichrome staining, 40-fold).
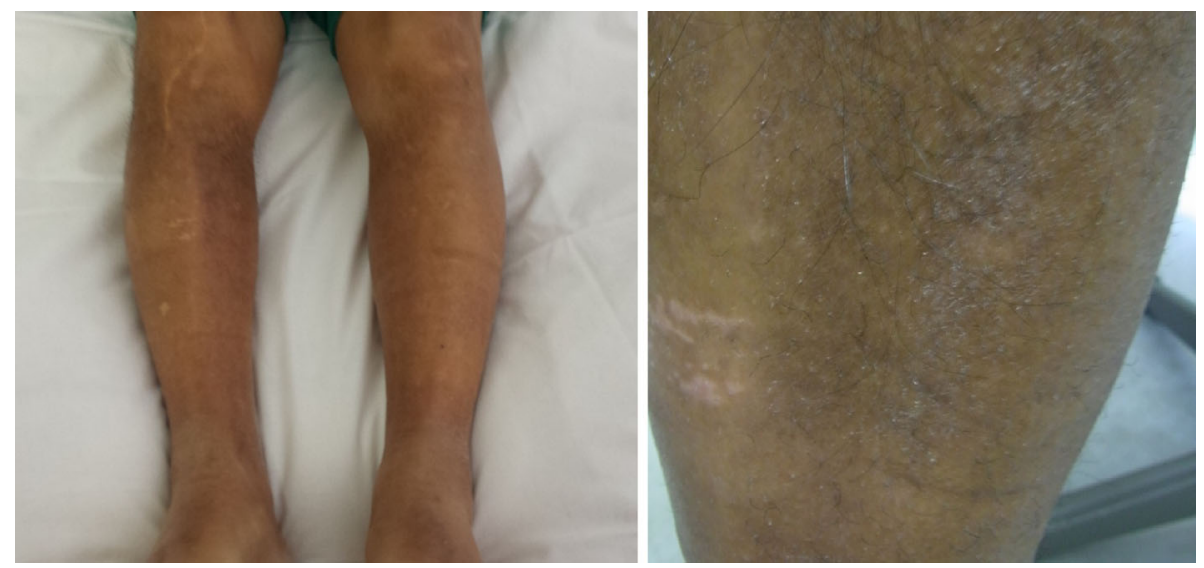

Figure 4. His lower limbs showed the typical finding of EF: limb edema with swelling and dimpling, referred to as an "orange-peel-like appearance". EF: eosinophilic fasciitis 


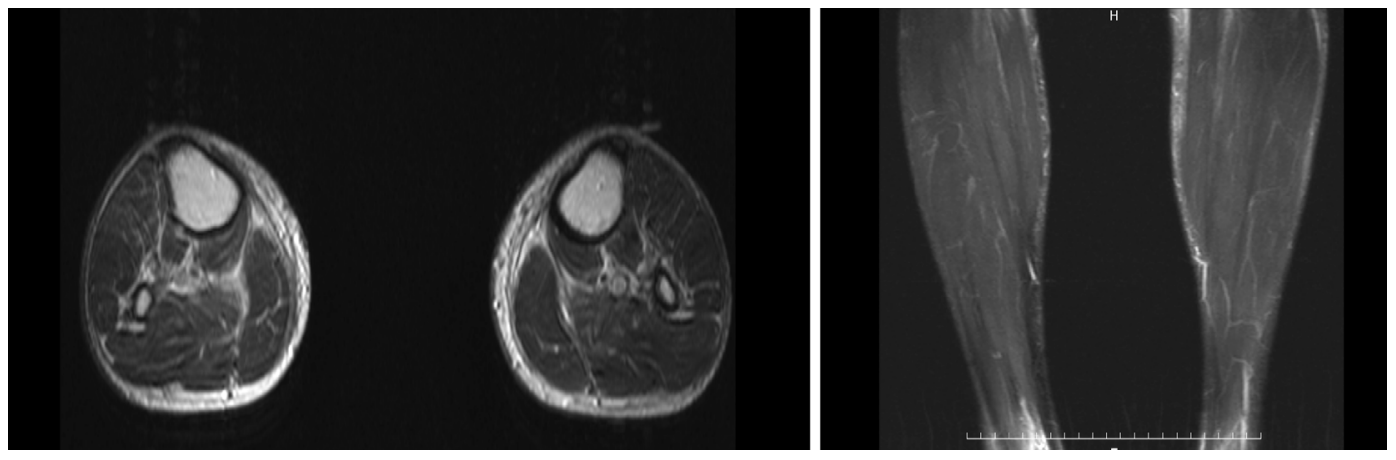

Figure 5. MRI of the lower limbs showed thickening of the fascia and high intensity of the fascia on fat-suppressed T2-weighted imaging.

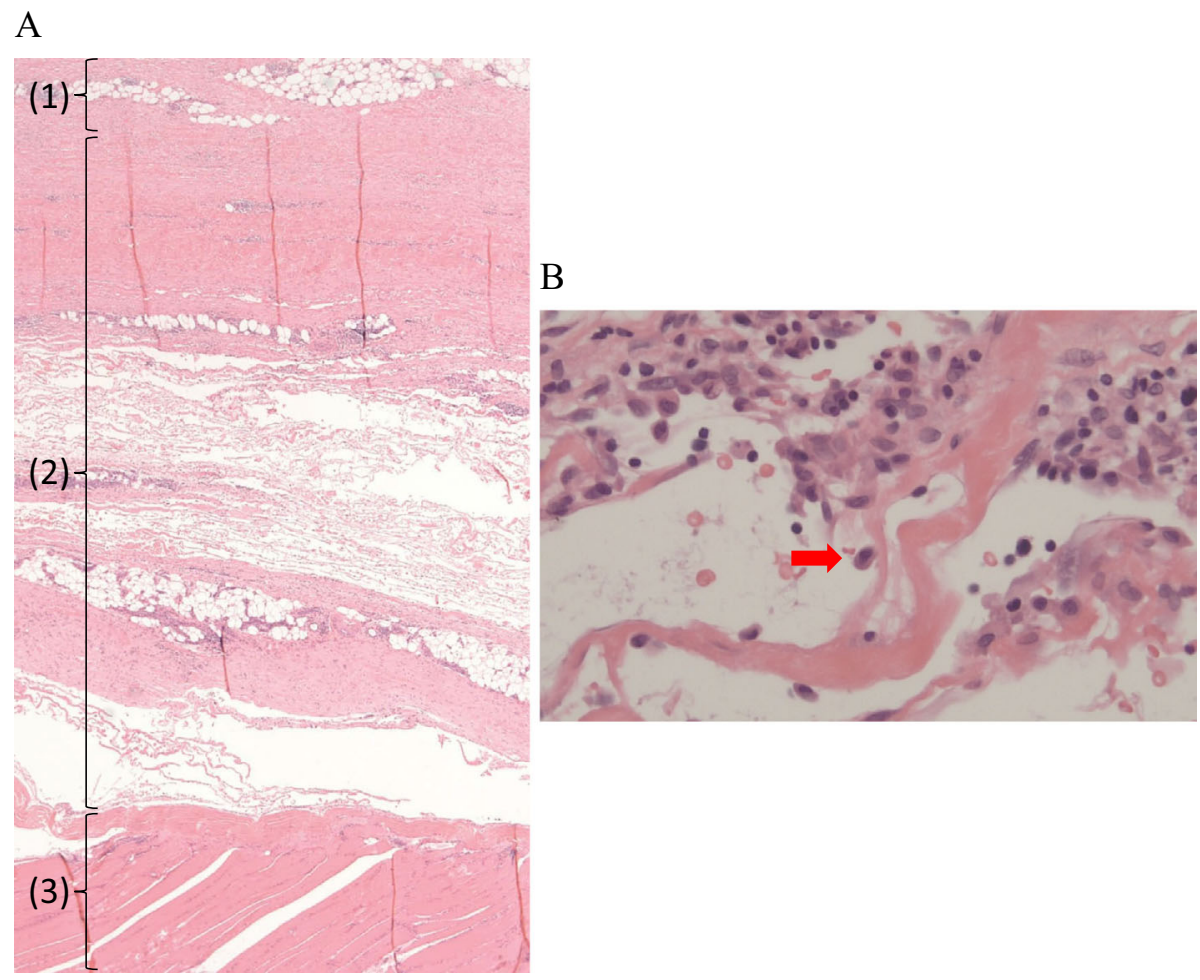

Figure 6. (A) An en bloc biopsy specimen showed thickening and edema of the fascia and hyperplasia and degeneration of the collagenous fiber (2). The muscle (3) and subcutaneous tissue (1) were normal [Hematoxylin and Eosin (H\&E) staining 10-fold]. (B) Inflammatory cell infiltration including eosinophils in the fascia (H\&E staining 400-fold). The arrow indicates eosinophil infiltration.

autoimmune disorder $(6,8)$. Similar to $\mathrm{AIH}, \mathrm{EF}$ is also known to be complicated by other autoimmune diseases, such as localized scleroderma, autoimmune thyroiditis, systemic lupus erythematosus, and rheumatoid arthritis (9-11). $\mathrm{AIH}$ and EF are believed to have similar autoimmune pathogeneses and complications with other autoimmune diseases. Therefore, it is plausible that AIH could be complicated by EF. However, AIH complicated by EF has never been reported, making the present case the first report of AIH complicated by EF.

EF complicated by AIH is not easily detectable. The characteristic physical finding of EF is edema of the limbs with swelling and dimpling, referred to as an "orange-peel-like appearance," or depression of skin along superficial veins, known as a "groove sign" $(7,12)$. However, since these characteristic physical findings are not found in all patients, and given that an "orange-peel-like appearance" is found more frequently in cases with long-standing disease (7), diagnosing EF at an early stage is not easy for general physicians. In addition, edema of the limbs is sometimes seen in hypoalbuminemia with chronic inflammatory disease or chronic hepatic disease. EF is thus not easily recognized by general physicians, especially if the typical physical findings are not obvious. Instead, EF might be mistaken for edema of the limbs resulting from the hypoalbuminemia associated with chronic hepatic disease. The onset of EF in the present case may have been at his first visit, which was earlier than the AIH onset. At his first visit, we failed to recognize the 


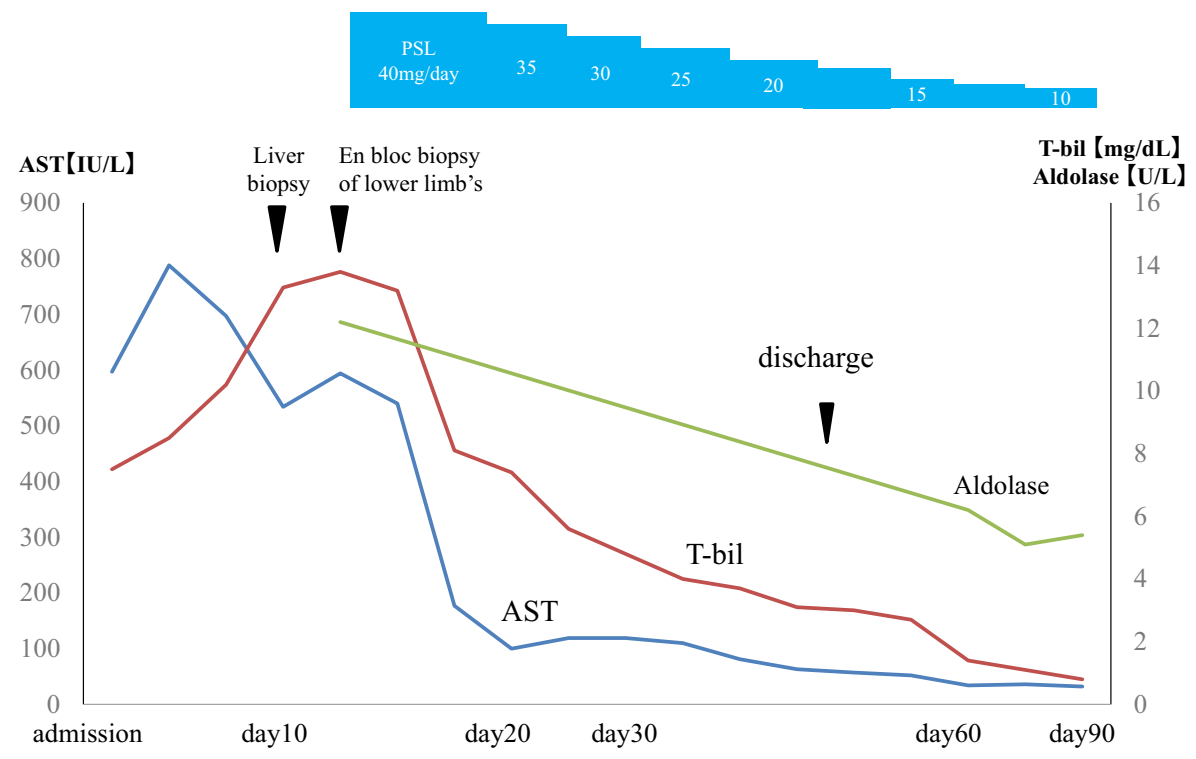

Figure 7. Clinical course.

physical findings of the legs, so we did not diagnose him with EF. However, after the diagnosis of AIH was made, we explored the possibility of complication with other autoimmune diseases and were aware of the typical physical findings of EF. Then, in conjunction with the MRI findings from the lower limbs and the en bloc biopsy findings, we finally diagnosed him with EF.

Many previous reports have reported that PSL was effective for the treatment of EF $(11,13)$. Therefore, even if EF had not been diagnosed, it would still have been improved by the PSL used to treat the AIH, and unfortunately, the EF might not have been diagnosed. However, because the response to steroid therapy is not always the same between AIH and EF, we should assess the etiology of edema in the legs at the initial diagnosis objectively.

Although no cases of AIH complicated by EF have been previously reported, some cases of chronic liver disease complicated by EF have been described (14-19). Loeliger et al. (14) reported a case of reactive hepatitis complicated by EF. In that report, the hepatitis might have been $\mathrm{AIH}$, given the high IgG levels. In addition, Tahara et al. (15) reported a case of primary biliary cholangitis complicated by EF. In a Brazilian and Japanese report, a patient with chronic hepatitis $\mathrm{C}$ was complicated by $\mathrm{EF}(18,19)$. Hepatitis $\mathrm{C}$ viral infection is now known to induce several extrahepatic diseases, including autoimmune diseases (20). As mentioned above, the pathological mechanism is not yet clear, but it is feasible for autoimmune liver disease to be complicated by EF. However, EF is not recognized well by physicians, gastroenterologists, and hepatologists. Therefore, many cases of EF might be missed. Although the frequency of EF complicated by AIH is not clear, further reports should be accumulated.

In the present patient, the M2BPGi level was reduced immediately along with the ALT decrease after steroid therapy. M2BPGi is known to reveal both inflammation and fibrosis associated with chronic liver disease, including AIH (21). In general, liver fibrosis can take a relatively long time to improve after PSL treatment. Therefore, the immediate decrease in M2BPGi observed in the present case might have been due to the amelioration of liver inflammation. In addition, aldolase, a biomarker of EF, was reduced after PSL treatment along with the leg edema disappearance. In a previous report, aldolase was found to reflect the disease activity of EF more accurately than eosinophils and the erythrocyte sedimentation rate (22). We should not only check for the presence of leg edema but also assess the aldolase level after PSL therapy for patients with EF.

In conclusion, AIH can be complicated by EF, and these diseases are improved drastically by administration of PSL. Some cases of EF complicated by AIH may remain unrecognized; therefore, we should carefully evaluate the physical findings, including the appearance of the legs, in order to detect other coexisting autoimmune diseases when we make a diagnosis of AIH.

The authors state that they have no Conflict of Interest (COI).

\section{References}

1. Abe M, Mashiba T, Zeniya M, Yamamoto K, Onji M, Tsubouchi $\mathrm{H}$; Autoimmune Hepatitis Study Group-Subgroup of the Intractable Hepato-Biliary Disease Study Group in Japan. Present status of autoimmune hepatitis in Japan: a nationwide survey. J Gastroenterol 46: 1136-1141, 2011.

2. Shulman LE. Diffuse fasciitis with hypergammaglobulinemia and eosinophilia: a new syndrome? J Rheumatol I: 46, 1974.

3. Bischoff L, Derk C. Eosinophilic fasciitis: demographics, disease pattern and response to treatment: report of 12 cases and review of the literature. Int J Dermatol 47: 29-35, 2008.

4. Czaja AJ. Autoimmune hepatitis. Part A: pathogenesis. Expert Rev Gastroenterol Hepatol 1: 113-128, 2007.

5. Liberal R, Serena M, Mieli-Vergani G, Vergani D. Pathogenesis of autoimmune hepatitis. Best Pract Res Clin Gastroenterol 25: 653- 
664, 2011.

6. Seibold JR, Rodnan GP, Medsger TA Jr, Winkelstein A. Circulating immune complexes in eosinophilic fasciitis. Arthritis Rheum 25: 1180-1188, 1982.

7. Berianu F, Cohen M, Abril A, Ginsburg WW. Eosinophilic fasciitis: clinical characteristics and response to methotrexate. Int $\mathrm{J}$ Rheum Dis 18: 91-98, 2015.

8. Kent L, Cramer S, Moskowitz R. Eosinophilic fasciitis: clinical, laboratory, and microscopic considerations. Arthritis Rheum 24: 677-683, 1981.

9. Lebeaux D, Sene D. Eosinophilic fasciitis (Shulman syndrome). Best Pract Res Clin Rheumatol 26: 449-458, 2012.

10. de Masson A, Bouaziz JD, Peffault de Latour R, et al. Severe aplastic anemia associated with eosinophilic fasciitis: report of 4 cases and review of the literature. Medicine (Baltimore) 92: 69-81, 2013.

11. Lakhanpal S, Ginsburg WW, Michet CJ, Doyle JA, Moore SB. Eosinophilic fasciitis: clinical spectrum and therapeutic response in 52 cases. Semin Arthritis Rheum 17: 221-231, 1988.

12. Lebeaux D, Francès $C$, Barete $S$, et al. Eosinophilic fasciitis (Shulman disease): new insights into the therapeutic management from a series of 34 patients. Rheumatology (Oxford) 51: 557-561, 2012.

13. Endo Y, Tamura A, Matsushima Y, et al. Eosinophilic fasciitis: report of two cases and a systematic review of the literature dealing with clinical variables that predict outcome. Clin Rheumatol 26: 1445-1451, 2007.

14. Loeliger AE, Van Den Brink HR, Derksen RHWM, Bijlsma JWJ. Eosinophilic fasciitis presenting with a reactive hepatitis. Clin Rheumatol 10: 440-444, 1991.

15. Tahara K, Yukawa S, Shoji A, Hayashi H, Tsuboi N. Long-term remission by cyclosporine in a patient with eosinophilic fasciitis associated with primary biliary cirrhosis. Clin Rheumatol 27: 1199-1201, 2008.

16. Jacobs MB. Eosinophilic fasciitis, reactive hepatitis, and splenomegaly. Arch Intern Med 145: 162-163, 1985.

17. Murata K, Endo S, Tsukifuji R, Shinkai H. A case of eosinophilic fasciitis. Rinsho derma (Tokyo) 42: 514-515, 2000 (in Japanese).

18. Shibata M, Miyatsuka T, Nishida T, et al. A case report of eosinophilic fasciitis associated with decompensated type C cirrhosis. Med J Osaka Prefectural General Hospital 22: 53-55, 1999 (in Japanese).

19. Shinjo SK, de Souza FHC. Eosinophilic fasciitis in a patient with hepatitis $\mathrm{C}$ virus infection: coincidence or association? Rheumatica Acta: Open Access 1: 1-3, 2017.

20. Negro F. Facts and fictions of $\mathrm{HCV}$ and comorbidities: steatosis, diabetes mellitus, and cardiovascular diseases. J Hepatol 61: 6978,2014

21. Nishikawa $\mathrm{H}$, Enomoto $\mathrm{H}$, Iwata $\mathrm{Y}$, et al. Clinical significance of serum Wisteria floribunda agglutinin positive Mac-2-binding protein level and high-sensitivity C-reactive protein concentration in autoimmune hepatitis. Hepatol Res 46: 613-621, 2016.

22. Nashel J, Steen V. The use of an elevated aldolase in diagnosing and managing eosinophilic faciitis. Clin Rheumatol 34: 1481$1484,2015$.

The Internal Medicine is an Open Access journal distributed under the Creative Commons Attribution-NonCommercial-NoDerivatives 4.0 International License. To view the details of this license, please visit (https://creativecommons.org/licenses/ by-nc-nd/4.0/).

(C) 2019 The Japanese Society of Internal Medicine Intern Med 58: 667-673, 2019 